\title{
Agôn
}

Revue des arts de la scène

7| 2015

La Distribution

\section{La distribution comme phénomène pop : l'antiteater de Rainer Werner Fassbinder et la Needcompany de Jan Lauwers}

\section{Stéphane Hervé}

\section{(2) OpenEdition \\ Journals}

Édition électronique

URL : http://journals.openedition.org/agon/3183

DOI : 10.4000/agon.3183

ISSN : 1961-8581

Éditeur

Association Agôn

Référence électronique

Stéphane Hervé, "La distribution comme phénomène pop : l'antiteater de Rainer Werner Fassbinder et la Needcompany de Jan Lauwers ", Agôn [En ligne], 7| 2015, mis en ligne le 11 juillet 2015, consulté le 20 avril 2019. URL : http://journals.openedition.org/agon/3183; DOI : 10.4000/agon.3183

Ce document a été généré automatiquement le 20 avril 2019.

Association Agôn et les auteurs des articles 


\title{
La distribution comme phénomène pop : l'antiteater de Rainer Werner Fassbinder et la Needcompany de Jan Lauwers
}

\author{
Stéphane Hervé
}

1 Rapprocher les pratiques théâtrales de Rainer Werner Fassbinder et de Jan Lauwers n'a a priori rien d'évident, tant celles-ci s'inscrivent dans des contextes géographiques, historiques et politiques bien différents, et tant que leurs esthétiques semblent éloignées ${ }^{1}$ . Pourtant, la trajectoire personnelle de ces deux artistes présente des similitudes trajectoire qui, comme nous allons le voir, engage un rapport à la distribution présentant, lui aussi, plusieurs points de convergence. Tous deux débutent leur carrière théâtrale par la pratique de la création collective : Fassbinder participe aux mises en scène collégiales de l'Action-Theater dès $1967^{2}$, Jan Lauwers fonde avec d'autres artistes en 1981, à Anvers, le collectif au nom programmatique de Epigonen zonder leiding van, c'est-à-dire «Epigonen, sous la direction de personne ». Ils fondent ensuite un nouveau collectif, respectivement l'antiteater (1969) et la Needcompany (1987), dont ils deviennent le centre névralgique en assumant les fonctions d'auteur et de metteur en scène principal ${ }^{3}$. De fait, ils écrivent leurs textes théâtraux et les mettent en scène (ainsi que d'autres textes) pour un ensemble constitué autour d'eux : même si les contours de cet ensemble demeurent flous ${ }^{4}$, ils écrivent donc pour des performers singuliers.

2 Lorsque Jan Lauwers déclare qu'il ne peut écrire " que pour des gens qui l'inspirent ${ }^{5}$ et qu'il a choisi de se consacrer pleinement au théâtre parce qu'il aime "participer à des processus collectifs " ${ }^{6}$, lorsque Fassbinder confie qu'il a "toujours écrit pour le théâtre avec l'objectif précis de travailler avec un groupe $»^{7}$, sans lequel il ne peut envisager de faire des mises en scène ${ }^{8}$, ces deux artistes ne font pas preuve d'originalité au regard de l'histoire du théâtre. En effet, la transitivité de l'écriture théâtrale ou la collaboration durable d'un metteur en scène avec un ensemble d'acteurs, ne sont pas des phénomènes nouveaux, propres à l'époque contemporaine. L'histoire du théâtre abonde en exemples 
d'auteur-directeur de troupe-metteur en scène qui écrivent ou travaillent pour un ensemble de comédiens déjà constitué, dont le plus emblématique serait sans doute Molière. Seulement, il apparaît que le paradigme qui soutenait cette écriture transitive et collective a changé. Le système dramaturgique des types, qui présidait à la fois à la constitution de l'ensemble et aux choix dramaturgiques de l'auteur, qui permettait de réguler l'attribution des rôles au sein de la troupe en fonction d'emplois spécialisés et d'une organisation hiérarchique, a été fortement remis en cause depuis le début du $\mathrm{xx}^{\mathrm{e}}$ siècle et n'est plus en vigueur aujourd'hui. Les collectifs théâtraux se constituent maintenant avant tout selon des affinités intersubjectives (formation commune, phénomène de cooptation...), et le partage d'un objectif esthétique (c'est le cas de la Needcompany) ou d'un dessein politique (celui de l'antiteater). Or, les valeurs qui régissent la constitution de ces collectifs excluent, il nous semble, l'idée de distribution, et les connotations de sélection, de hiérarchisation, de spécialisation, voire de talent spécifique, qui lui sont afférentes. Le refus des processus distributifs au sein d'un collectif affecte également le passage à la scène : il s'agit de trouver les formes plus idoines à la mise en place d'un ethos scénique collectif. La scène est alors le lieu de l'autoreprésentation du collectif théâtral, dans une perspective politique. Il s'agit de donner à voir et à entendre un agencement communautaire exemplaire, en rupture avec l'atomisation individualiste de la société, une forme expérimentale de collaboration et de cohabitation, d'être-ensemble.

\section{L'exigence égalitariste}

3 Pour conjurer ou minorer les rapports de pouvoir relatifs à la distribution, les collectifs des années 1960 eurent recours à diverses stratégies : abandon des rôles (Living Theatre), permutation des rôles (Théâtre du Soleil), distribution de plusieurs acteurs pour un même rôle... La mise en scène d'Antigone par l'Action-Theater, dans laquelle s'intègre Fassbinder en 1967, est symptomatique de ce dernier procédé. Étant données les aspirations égalitaristes du collectif, il fut décidé d'attribuer le rôle de la protagoniste qui, par son omniprésence et les valeurs que ce rôle véhicule, domine l'économie dramatique de l'adaptation réalisée par Peer Raben, aux quatre personnalités féminines du groupe, ce pour déjouer toute possibilité de hiérarchisation De plus, le choix de placer sur ces scènes des individus proches du mouvement hippie, sans lien avec le théâtre, en tant que figurants, témoigne également de la volonté de sortir du système distributif, en montrant que le groupe sur scène est avant tout une communauté de vie et d'idées, et non un collectif spécialisé dans le théâtre. Il s'agissait donc par là de rompre avec l'enseignement pratique, relativement traditionnel qui venait d'être dispensé aux membres de l'ActionTheater dans des académies munichoises, et d'exhiber que la présence scénique n'est pas la conséquence d'une capacité technique, mais d'un choix existentiel.

Comme le montre l'exemple précédent, l'ethos scénique collectif impose souvent une exigence égalitariste : il ne doit pas y avoir de hiérarchie dans le collectif, non seulement sur scène, mais aussi idéalement dès le moment de l'écriture des pièces. Ainsi les textes de Fassbinder, de Katzelmacher jusqu'au Village en flammes, comportent un nombre considérable de personnages, auxquels est attribué un nombre à peu près similaire de répliques. En résulte une absence de rôles principaux revendiquée, comme en témoigne cette remarque rétrospective de Fassbinder : «Nous nous étions mis d'accord qu'il serait bon que tous les rôles soient à peu près équivalents $»^{9}$. Si une telle affirmation de principe 
n'est pas repérable dans la bouche de Lauwers, cet extrait d'une réplique tenue par un personnage de La Maison des cerfs, qui décrit le fonctionnement de son cercle familial, pourrait valoir comme programme du fonctionnement de la Needcompany : « Mais tout le monde avait à faire, ici à la maison des cerfs, et les tâches étaient divisées équitablement $\aleph^{10}$. En effet, comme pour l'antiteater, il y a une équivalence relative du nombre de répliques accordées à chacun des personnages, et partant à chacun des performers. Même dans une pièce centrée autour d'un seul personnage, comme La Chambre d'Isabella, tous les personnages ont droit à un monologue.

5 L'ethos scénique collectif se traduira également par la présence continuelle de l'ensemble de la distribution sur la scène. L'antiteater est ainsi en permanence sur scène, même si l'intrigue suppose une sortie des personnages incarnés. Il y a certes une explication matérielle et pragmatique à un tel choix, à savoir: l'absence de dégagements et de coulisses, l'étroitesse de l'arrière-salle de la Witwe Bolte, dans laquelle l'antiteater présentait ses productions sur une estrade de huit mètres carrés. Seulement, Fassbinder revendique ce choix comme une prise de position esthétique et politique, et l'explique ainsi :

Depuis le début, il était évident que, dans une telle situation, où on ne gagnait pas beaucoup d'argent, où on faisait surtout du théâtre par plaisir et par désir d'appartenir à un groupe, il ne pouvait y avoir de décalage entre la représentation scénique de l'ensemble et la réalité. [...] Cela s'est cristallisé dans une forme que j'ai observée encore longtemps après : tous les membres restaient toujours sur la scène, pour qu'ils puissent y être au moins le même temps. ${ }^{11}$

De la même manière, il n'y a pas de sortie de scène dans les productions de Jan Lauwers, puisqu'il s'agit de montrer scéniquement une figuration du collectif. D'ailleurs, cette prescription explique en partie une caractéristique essentielle de la dramaturgie de l'auteur belge : la coprésence des morts et des vivants. Si ses pièces semblent hantées par la question du deuil, par le problème de la cohabitation entre vivants, et entre vivants et morts, ces enjeux existentiels portés par les textes correspondent également à une conviction esthétique, touchant à une manière d'être-scénique : le performer ne meurt pas sur scène (c'est son personnage), et comme il reste sur scène, il doit continuer à interagir.

7 La présence continuelle des performers sur scène, leur disposition, leur espacement, leurs contacts, leur rapprochement, l'entremêlement ou l'isolement des voix, dessinent indubitablement des images communautaires, que la clôture de la scène élève au statut de modèles, et que la dramaturgie transpose dans l'ordre de la fiction narrative. De fait, les deux œuvres abondent en de telles images : chez Fassbinder, communautés villageoises ( Le Bouc, Le Village en flammes, voire Le Café), révolutionnaires (Anarchie en Bavière, Die Bettleroper), occasionnelles ( $\mathrm{u} u$ Sang sur le cou du chat); chez Lauwers, communautés villageoises également (Place du marché 76), familiales (Le Bazar du homard, La Maison des cerfs), théâtrales (La Maison des cerfs).

8 Il faut toutefois relativiser les incidences réelles de cette ambition égalitaire. Les fluctuations de la constitution des collectifs s'accompagnent en effet souvent d'une remise en cause du principe égalitaire et d'une reconduction d'un certain système des emplois, basé sur des liens d'intelligence artistiques ou relationnels. Fassbinder dérogera ainsi à ce principe d'égalité après la rencontre avec Margit Carstensen à Brême, qui deviendra son égérie théâtrale et interprétera des grandes figures féminines (Hedda Gabler, la Julie de Strindberg). Fassbinder infléchira alors son esthétique dramatique pour 
placer au centre un personnage féminin (Phoebe dans Du Sang sur le cou du chat, et surtout Geesche dans Liberté à Brême, Petra dans Les Larmes amères de Petra von Kant), Ainsi, l'arrivée de Carstensen au sein de la bande de l'antiteater, bouleverse totalement la pratique théâtrale de Fassbinder: il reconnaît en elle une comédienne qui, grâce à ses capacités techniques, peut incarner les affects qu'il cherche à explorer (la panique névrotique, la joie perverse, l'hystérie), et ce d'autant plus que Carstensen, dès le début, profite de l'esthétique du metteur en scène munichois, avec laquelle elle s'accorde, pour relancer sa carrière théâtrale.

$9 \quad$ L'intégration de nouveaux membres a pu réinstaurer également une certaine hiérarchie au sein de la Needcompany, du fait d'un certain manque de connaissance réciproque (et incidemment de connivence) entre l'auteur et le performer. Ainsi, Lauwers attribue un nombre moindre de répliques aux "jeunes" membres de la Needcompany, souvent danseurs, dans la trilogie Sad Face/Happy Face ${ }^{12}$. La première partie de La Maison des cerfs donne même une représentation dramatisée de l'entrée au sein du collectif: durant cette séquence, intitulée "Les Loges ", les performers mènent en leur propre nom une scène de discussion qui s'achève sur le rejet du personnage de Yumiko Yanaka (danseuse arrivée récemment au sein du collectif), que les autres ne reconnaissent pas et qu'ils soupçonnent d'avoir volé un téléphone portable. Si ce rejet peut être assurément perçu comme une métaphore dénonçant l'exclusion des immigrés, il demeure qu'il pointe également l'impossible égalité entre les membres de la compagnie, le nouvel arrivant ayant de fait moins de liens intimes avec les autres performers.

À l'inverse, les compagnons de longue date, Viviane de Muynck et Dirk Roofhooth qui ont collaboré avec Lauwers dès les années 1990, ont été placés au centre de deux spectacles récents, respectivement $L a$ Chambre d'Isabella ${ }^{13}$ et $L^{\prime}$ 'Art du divertissement. Il ne peut pas être question de vedettariat dans les deux cas, car, même si les deux comédiens en question mènent une carrière remarquable en dehors de la Needcompany sur les scènes flamandes (et à la télévision et au cinéma), leur notoriété médiatique reste limitée. C'est davantage la durée du travail commun, et donc leur (re)connaissance réciproque, qui incite l'auteur à développer davantage leur rôle. D'ailleurs, il est significatif que les trois danseurs (ceux de la création ou ceux des nombreuses reprises), auxquels aucun rôle n'était attribué dans le texte de La Chambre d'Isabella (ils étaient présentés par Lauwers au début du spectacle comme les deux hémisphères du cerveau et les zones érogènes de l'héroïne), acquièrent de plus en plus d'importance dramatiquement, au regard du nombre des répliques accordées dans les pièces suivantes.

\section{La place de l'auteur}

11 Le principe d'égalité est, de toute façon, mis à mal par la position qu'occupent Fassbinder et Lauwers au sein de leur collectif respectif, à savoir celle de fondateur, auteur et metteur en scène, et incidemment de leader ${ }^{14}$. L'intéressant est de constater que ces deux artistes, à travers le rôle, dramatique ou performatif, qu'ils s'attribuent lorsqu'ils apparaissent sur scène, questionnent leur relation ambiguë au collectif, entre appartenance et souveraineté individuelle., En effet, les rôles dans lesquels Fassbinder et Lauwers se distribuent sont toujours singuliers : sur scène,, ils ne s'effacent pas dans le collectif, mais occupent une position scénique problématique, au sens où elle problématise les rapports de pouvoir - antinomiques à l'idée de collectif, comme à celle 
de cet ethos scénique collectif, dont nous avons parlé - qui lient l'auteur-metteur en scène au reste de l'ensemble.

Il n'est pas anodin, en effet, que les rôles que Fassbinder s'attribue lui-même dans les mises en scène de ses propres textes relèvent de la catégorie de l'outsider, rejeté par la communauté : Jorgos, dans Le Bouc est un immigré grec arrivant dans un village bavarois, bientôt objet des préjugés racistes des membres de la jeunesse locale désœuvrée, réduit au rôle de victime expiatoire; dans Die Bettleroper, réécriture contre-culturelle de l'Opéra des gueux de John Gay, Mecki est un genre de hippie aspirant à l'oisiveté totale, étranger et indifférent à la circulation sociale des transactions financières et affective, et pour cela même, objet de désir et de répulsion, qu'il faudra expulser de l'espace partagé pour que les trafics qui l'animent puissent survivre. Dans les deux cas, Fassbinder semble s'identifier à la place du bouc émissaire, comme s'il s'agissait de thématiser les relations conflictuelles entre l'auteur/metteur en scène et le collectif ${ }^{15}$. Bien plus, le laconisme de Mecki, l'incapacité langagière de Jorgos indiquent qu'il est possible de voir dans ces dramaturgies violentes une représentation symbolique des enjeux esthétiques latents à l'œuvre dans l'exercice de la distribution, comprise comme délégation : écrire pour un collectif, pour une communauté, c'est effacer sa propre parole, c'est s'offrir, plus ou moins violemment, aux autres.

13 Il n'est pas possible de trouver de figures dramatiques équivalentes chez Lauwers : l' outsider rejeté ou sacrifié ne peut faire office d'image de l'auteur, en ce qu'il diverge trop fortement de la personnalité de l'auteur. En revanche, Lauwers s'est essayé à mettre en scène cette délégation, sous un mode beaucoup moins âpre, plus décontracté, dans sa production de La Chambre d'Isabella. L'artiste, vêtu d'un costume blanc tapageur, accueille avec bienveillance les spectateurs, présente le spectacle, les différents objets présents sur scène, issus de la collection de son père récemment défunt, les différents membres de la compagnie et leur rôle dans l'action de la pièce, avant de se mettre à l'écart la plupart du temps, assis sur une chaise en marge de la scène. Ensuite, il reste dans une position d'observateur, non sans réaction (il sourit aux différents numéros), du public et des acteurs durant toute la représentation, sauf lorsqu'il attrape un accessoire pour aider les performers et participe à la présentation de la collection. Enfin, il joue de la guitare lors du dernier morceau, joué et chanté collectivement ${ }^{16}$. Lauwers s'est expliqué sur sa présence scénique, alors même qu'il n'incarnait aucun des rôles prévus par le texte, en donnant deux raisons, apparemment contradictoires. D'une part, il voulait montrer par ce procédé la dépossession de l'artiste à l'œuvre lors du passage à la scène : « On pourrait dire que le simple fait que je sois sur scène, sans prendre part à l'action, rend évident que celle-ci ne me concerne plus $»^{17}$. D'autre part, il espérait proposer un discours critique, en rendant sa présence superficielle et improductive, sur la tendance observable, selon lui, dans le champ de l'art contemporain, à savoir la personnalisation excessive de la production esthétique : «Dans ce spectacle, j'étais moi aussi sur scène, vêtu d'un costume blanc. Je voulais susciter une réflexion sur le fait que de nos jours, on se focalise davantage sur l'artiste que sur l'art. Mais je pense que cela n'a pas vraiment été compris » ${ }^{18}$. De fait, l'artiste flamand regrette que le spectacle fût généralement compris sous un angle autobiographique, ce qu'il n'est que partiellement ou seulement à l'origine.

En fait, Jan Lauwers ne tint pas compte de l'ambivalence paradoxale de la dépossession de l'auteur individuel à travers la distribution collective, mise au jour par Boris Groys. D'après le philosophe allemand, la dépossession de soi dans l'œuvre collective s'accompagne nécessairement du renforcement de l'auctorialité et de la personnalisation 
de l'œuvre, du fait que cette dépossession est toujours incomplète et qu'elle « est toujours formulée par l'auteur $\aleph^{19}$. Il faut alors comprendre que l'écriture pour un collectif suppose à la fois un effacement de l'auteur et une accentuation de la personnalisation de l'œuvre, double élan propre au régime esthétique contemporain, à la manière d'un Warhol.

\section{Une écriture contrainte et transitive}

15 La mention de Warhol, tout étonnante qu'elle soit dans un développement consacré aux processus de distribution dans un collectif théâtral, est justifiée par l'aveu de Lauwers au sujet d'une similitude certaine entre les processus de création cinématographique de l'artiste américain et ses propres modes de production :

«Lorsqu'on demandait à Warhol si tous ces gens qui gravitaient autour de lui ne le dérangeaient pas, il répondait que ce n'étaient pas les gens qui étaient autour de lui, mais lui autour des gens. Je peux parfaitement comprendre cette réponse $»^{20}$.

De fait, si la démarche de Lauwers ne peut être comparée au parasitisme de Warhol, qui faisait des «inadaptés qui venaient à la Factory [des] sources d'inspiration ${ }^{21}$, il n'en reste pas moins que l'œuvre théâtrale récente de Lauwers emprunte aux individualités de la Needcompany dans la construction de figures fictives ou de situations narratives. De fait, l'ethos scénique collectif possède une double dimension: à la constellation de positions dramatiques ou de fonctions performatives se superpose une autoprésentation du collectif en tant qu'ensemble d'individualités. En d'autres termes, dans cet ethos scénique, les régimes de présence des performers ne doivent pas seulement laisser accroire à une égalité des rôles, mais également faire advenir un effet d'authenticité subjective, ce que révèle assez explicitement cette déclaration de Jan Lauwers : «Pour moi, un acteur est un performeur qui présente un personnage tout en étant lui-même $»^{22}$. Il ne s'agit pas, par là, d'aboutir à un effet de distanciation brechtien, mais de montrer que l'idée de collectif reposerait également sur le respect de l'individualité de chacun des performers.

17 L'exigence de l'ethos scénique collectif engage par conséquent la question de la transitivité de l'écriture théâtrale pour un ensemble déjà constitué. Si les deux auteurs proclament que l'écriture est avant tout un acte solitaire, il reste que l'écriture est dans un rapport de dépendance à la constitution du collectif. Jan Lauwers affirme, par exemple, que le choix des acteurs est le préalable du processus d'écriture : «le choix des acteurs est très important. Je ne peux commencer à écrire que quand j'ai un ensemble. La première phase de mon travail est de choisir des gens $»^{23}$. En d'autres termes, la distribution précède le processus créatif, ou plus précisément, elle en est l'étape initiale, si par distribution nous entendons ici la formation d'un groupe de performers en vue de la production d'une œuvre scénique, et non l'attribution de rôles préétablis. Selon Lauwers, ce choix ne dépend pas d'une possible adéquation à une idée de rôle, ni même de la spécialisation des individus choisis (musique, danse, théâtre). L'artiste flamand sélectionnerait ses performers selon leur aspiration à "une liberté inconditionnelle", c'est-à-dire leur propension à mettre toute leur personne en jeu au-delà des normes sociales et de bienséance ${ }^{24}$.

18 L'écriture prend en compte, alors, les caractéristiques de chacun des performers. Pour donner un exemple évident, l'arrivée des danseuses Fumiko Yanaka et Sung-Im Her dans la Needcompany incitera Jan Lauwers à inclure dans le personnel dramatique de Place $d u$ Marché 76, deux personnages d'origine asiatique. De la même manière, les différences de 
générations entre les membres de la compagnie belge induisent des rapports interindividuels particuliers dans l'économie dramatique des pièces: la présence de noyaux familiaux au sein de chacun des textes récents de Jan Lauwers (à l'exception de L'Art du divertissement qui joue davantage sur les différences de niveau d'expérience sociale) s'explique assurément par l'obsession thématique de la filiation chez l'auteur belge, mais aussi tout simplement par la composition du collectif d'acteurs. En revanche, l'appartenance à une même génération des membres de l'antiteater limite la présence des motifs familiaux dans l'œuvre dramatique de Fassbinder. Bien plus, elle influence le choix de pièces d'autres auteurs: Fassbinder avoue qu'il s'est tourné vers les textes de Marieluise Fleißer plutôt que vers ceux d'Horváth, en raison de la trop grande différence d'âge entre les personnages de ceux-ci ${ }^{25}$. Katzelmacher (Le Bouc) rend manifeste cette contrainte par le choix d'une bande de jeunes villageois, ayant, on le suppose, l'âge de la bande qui entoure Fassbinder.

Il y aurait donc une porosité indéniable et explicite entre performer et rôle. L'asymptote de cette porosité serait atteinte dans La Maison des cerfs, pièce dans laquelle les dénominations des rôles correspondent aux noms des performers, et qui surtout a pour origine un événement réel qui vient bouleverser la troupe lors des représentations du Bazar du homard au Festival d'Avignon en 2006: «Tijen Lawton a appris que son frère, le journaliste Kerem Lawton, avait été tué au Kosovo. La mort tragique de Lawton est le point de départ d'un texte sur un groupe de gens de théâtre confrontés de façon de plus en plus directe à la dure réalité du monde qu'ils sillonnent ${ }^{26}$. La pièce débute alors au retour de la danseuse, partie chercher le corps de son frère dans le pays encore en guerre. Ainsi, Lauwers puise dans le vécu de sa collaboratrice pour construire son œuvre. Pour autant, il n'a de cesse de dénoncer cette démarche, dans les différents entretiens qu'il accorde depuis une dizaine d'années, en la qualifiant de narcissique. Seulement, le choix d'un événement personnel advenu à un des membres du collectif comme origine du processus de création (l'œuvre délaisse ensuite ce fondement factuel et ne cherche en aucun cas à être documentaire) est d'une certaine manière imposé par l'exigence d'autoprésentation scénique des individualités.

\section{La distribution comme constellation de personae}

Il est difficile de trouver dans l'œuvre théâtrale de Fassbinder une telle exploitation d'éléments biographiques factuels ${ }^{27}$. Pour autant, un phénomène nous interpelle à la lecture des différentes distributions des créations fassbinderiennes: la similitude des différents personnages incarnés par un même comédien. On remarque, par exemple, que Kurt Raab joue principalement des rôles caractérisés par une certaine perversité hystérique et tyrannique: Ubu dans Orgie Ubuh, le truand dépravé Peach dans Die Bettleroper, le serial-killer dans Preparadise sorry now, le boucher originellement masochiste dans Du Sang sur le cou du chat, le calomniateur malhonnête Maurizio dans Le Café. Les différentes figures féminines interprétées par Hanna Schygulla se présentent, quant à elles, comme des variations sur le thème de la jeune fille ingénue, sensible et amoureuse, mais aux aspirations bourgeoises, comme Polle dans Die Bettleroper ou la fille dans Du Sang sur le cou du chat. Ingrid Caven est pour sa part cantonnée à des rôles de vamps, de séductrices dominées par un fort appétit sexuel - d'Ingrid, qui se prostitue, dans Le Bouc, à Placida, prête à tout pour pouvoir à nouveau jouir dans les bras de son mari qui l'a délaissée dans Le Café, en passant par Peachie dans Die Bettleroper, cette femme 
qui prend du plaisir à être dominée par son mari. Nous pourrions continuer la liste en évoquant également les rôles de femmes sadiques d'Irm Herrmann, les personnages fraternels joués par Peer Raben, les incarnations mélancoliques d'Harry Baer, arrivé à la fin de l'aventure collective, ou encore les brutes généreuses dévolues à Rudolf Waldemar Brem. Cette constellation de rôles se répète donc de pièce en pièce (et de film en film), avec quelques aménagements nécessités par le sujet de l'œuvre. Les rôles dévolus à chacun des membres semblent proposer des variations autour des traits distinctifs d'une individualité singulière, qu'il est tentant de rapprocher de la notion classique de types théâtraux, voire même, si l'on reprend le lexique des moralistes, d'un caractère ${ }^{28}$. En fait, la constellation permet de créer des effets de reconnaissance chez le spectateur familier du théâtre fassbinderien, Elle s'appuie en fait sur l'individualité des acteurs, qui, par le fait des répétions/variations, deviennent des archétypes, et les rôles des avatars de ces archétypes.

21 Le même phénomène est à l'œuvre dans les dernières créations de la Needcompany (depuis La Chambre d'Isabella). On y retrouve en effet, d'un spectacle à l'autre, la répétition d'une même matrice individuelle qui connaît des inflexions selon sa fonction exercée dans l'économie dramatique de la pièce. Par exemple, les différents personnages interprétés par le comédien Benoît Gob se caractérisent par la combinaison d'une certaine animalité, d'une prédominance des instincts et des pulsions, inclinant au grotesque et à l'ivresse corporelle : l'alcoolique Arthur, tourmenté par son passé, dans $\mathrm{La}$ Chambre d'Isabella, le frustre et fidèle Vladimir dans Le Bazar du homard, le docteur aigri Dr. Joy dans L'Art du divertissement, le boucher violent et raciste de Place du marché 76. Des constats similaires peuvent être fait avec le reste de la distribution : Anneke Bonnema est une figure de femme dévouée et mélancolique, traversée par des failles affectives dans $L a$ Chambre d'Isabella et Place du Marché 76 ; Hans Peter Dahl, un homme mûr, velléitaire et enclin au retour sur soi dans les mêmes pièces et dans Le Bazar du homard; les différents avatars scéniques de Maarten Seghers se distinguent par leur spontanéité transgressive, par-delà bien et mal; Grace Ellen Barkey interprète souvent des femmes naïves, mais aussi suscitant un désir érotique ; et bien sûr, la figure emblématique de la Needcompany, Viviane de Muynck, offre son expérience pour dessiner des portraits de femme à la vitalité exacerbée et à la sagesse incontestable.

De fait, dans les créations de la Needcompany et de l'antiteater, le phénomène semble être le même: la récurrence des attitudes et comportements crée de forts effets de familiarité et un type individuel, commun à toutes ces occurrences par-delà les effetspersonnages ponctuels. Mais, les deux processus de distribution diffèrent dans le rapport à la personnalisation. Chez Fassbinder, la répétition de traits distinctifs assignés aux différentes apparitions scéniques d'un comédien n'est pas accompagnée d'indications explicites renvoyant à sa personnalité, alors que les pièces de Lauwers, à travers la dénomination des différents personnages, exhibent le lien qui les associe à l'individualité des performers. Cela est manifeste dans le texte de La Maison des cerfs, dans lequel les différents personnages portent le nom des performers (sans toutefois qu'il y ait coïncidence parfaite : les personnages ne sont pas les performers, même dans la première partie). De façon moins évidente, certains personnages de Place du marché 76 ont le même prénom que leurs interprètes: Benoît de Leersnyder (Benoît Gob) et Anneke De Leersnyder (Anneke Bonnema). Enfin, dans L'Art du divertissement, Viviane de Muynck joue le rôle de Liliane Van Muynck, sans compter Dirk Roofhooft, qui comme le sous-titre de l'œuvre l'indique (Needcompany joue la mort de Dirk Roofthooft), joue son propre rôle. 

présence scénique des membres de Needcompany, qui, «même lorsqu'ils agissent dans leur rôle", semblent évoluer " comme des personnes privées ${ }^{29}$. Ailleurs, le théoricien introduit, pour analyser cette pratique troublante et juguler la tentation de faire intervenir ici l'idée d'une authenticité subjective (le performer se montre en lui-même sur scène), la notion qui nous semble capitale de persona :

Se présentant et se mettant eux-mêmes en scène à l'intérieur du cadre du spectacle, ils ne sont plus totalement eux-mêmes; ils sont quelque chose comme leur propre persona - de la même manière qu'on parle dans les médias de la persona d'un comédien (entertainer). Par conséquent, les performers ne sont pas vraiment des acteurs, au sens où un acteur crée un rôle, et plus que des personnalités qui affichent une physicalité, une silhouette, une voix et une attitude singulière face à des spectateurs. ${ }^{30}$

L'allusion faite par Lehmann à la culture de masse de l'entertainment nous semble d'autant plus pertinente que la distribution des rôles, de fait, tient davantage à la formation d'une constellation de personae que d'une répartition selon le système théâtral des « emplois ». Dans les stars studies américaines, la notion de persona, est utilisée pour analyser la fusion d'éléments provenant de leurs rôles cinématographiques et de leur vie privée (relayés par les médias), qui déterminent l'image publique de la star ${ }^{31}$. En outre, la notion de persona semble capitale pour saisir ce qui se joue dans l'exhibition de soi, dans la forte personnalisation au sein de la culture pop. En suivant les analyses de Christophe Kihm sur la production de soi dans la sphère musicale rock, et en les transposant dans le champ théâtral,

L'authenticité se détermine dans le programme d'une réunion de la musique et de la vie. Ce que l'on joue dans la musique ou sur scène, dans la pop, c'est soi, sans aucune distance. Dans cette mise en scène de soi, dans cette fusion entre soi-même et soi dans la représentation, se joue le spectacle de l'authenticité. ${ }^{32}$

nous pourrions affirmer que les nouveaux modes d'apparition de la subjectivité sur les scènes contemporaines ressortissent à cette culture pop, basée sur la « fusion » entre une individualité et un rôle. D'ailleurs, ces analyses nous semblent davantage pertinentes pour saisir cette imbrication de la personnalité et du rôle que le modèle fréquemment invoqué de l'art de la performance. En effet, ce dernier suppose une auto-référentialité du sujet, empêchant toute écriture préalable et tout effet de figuration. Or, les processus de subjectivisation, repérables dans les productions de l'antiteater comme de la Needcompany, se développent dans un espace intermédiaire entre personnalité et rôle. Cet entre-deux, cet indéterminé subjectif engage alors la question de la distribution, puisqu'il prescrit des positions à l'intérieur du collectif, auxquelles il n'est possible de déroger qu'en niant l'individualité du performer. Or, s'il n'est pas question d'exhibition biographique, de vie privée chez Lauwers, il n'en demeure pas moins que l'écriture des rôles offre les conditions d'émergence de personae, puisque, comme le dit l'artiste flamand dans différentes interventions médiatique, celle-ci se fait «sur la peau » de ces performers, cette métaphore épidermique signifiant peut-être que l'écriture brouille leur personnalité, sans toutefois l'occulter ou la travestir ${ }^{33}$.

La persona se constitue donc à l'intersection d'un rôle et d'une individualité performative, elle se forge dans l'écriture par l'élaboration d'une figure, relativement indéterminée dramatiquement, à partir d'une singularité gestuelle, vocale, physique effective. Bien plus, la persona est ce qui ne cesse de revenir de rôle en rôle, comme noyau indéfectible, position à la fois virtuelle (dans un système dramatique lâche) et actuelle (dans 
l'occupation partagée de la scène), elle est ce reste essentiel qui transcende à la fois le personnage (celui-ci n'est que son support d'apparition) et le performer (son catalyseur). Ainsi, la notion de distribution au sein d'un collectif prend un nouveau sens : elle n'est pas seulement le postulat à partir duquel l'œuvre peut se déployer, elle est aussi la disposition des personae qui permet la cohésion narrative et la cohabitation spatiale. Distribuer, c'est consteller des personae, et par là-même, outrepasser les prescriptions d'égalité et d'indistinction intrinsèque au collectif, dans la formation d'un groupe. Nous pourrions donc dire que la distribution à l'œuvre dans l'écriture de Lauwers fait de la Needcompany un groupe, à en croire la définition qu'en donne Christophe Kihm, pour qui cette "entité première" de la culture pop, propose une unité qui «repose sur les bases d'une répartition précise des fonctions et des rôles $»^{34}$. Si le groupe pop s'avère être "la figure paradigmatique de l'art d'aujourd'hui $»^{35}$, c'est peut-être que sa forme collective suppose à la fois l'intrication entre rôle (fonction) et individualité et une répartition des positions au sein de cette forme. Bien plus, le groupe, issu de la culture pop, pourrait valoir comme forme hétérotopique sur les scènes théâtrales contemporaines, après les tentatives de communauté des années 1960-70, qui se sont rapidement confrontées à leurs propres apories, causées par la superposition d'un collectif théâtral et d'une communauté de vie, d'exigences artistiques et de relations intersubjectives exacerbées. Par là-même, la notion de distribution, dont nous avons dit qu'elle était antinomique de celle de collectif, retrouve une nouvelle pertinence pour décrire les formes communautaires scéniques: elle crée de l'accord à partir du présupposé de l'individualité singulière. Par la répartition narrative et scénique des personae, elle forge un ensemble collaboratif qui ne nie en aucune manière les différences individuelles.

La formation des personae fassbinderiennes ressortit à une autre logique, qui s'apparente à celle du star system. Influencé par son amour immodéré pour le cinéma américain, Fassbinder a procédé, dès ses débuts, à la métamorphose des membres de ces collectifs pour en faire des «stars ». À la manière, toujours, d'un Warhol qui aspirait à faire de la Factory un Hollywood en miniature, Fassbinder cherchait à créer les conditions de possibilité d'un Hollywood bavarois, même dans son travail théâtral. Fassbinder affirma plus tard qu'il avait considéré dès ses débuts ces acteurs " comme s'ils étaient des stars " ${ }^{36}$ . La relation à Hanna Schygulla est à ce propos emblématique. La jeune femme ne faisait pas partie du collectif à ses débuts, mais fréquentait le même cours dramatique que Fassbinder. Suite à l'incapacité de Marite Greiselis à tenir son rôle dans Antigone, Fassbinder, pourtant récemment intégré au collectif, réussit à imposer sa camarade pour faire partie de l'ensemble féminin incarnant la rebelle mythique. Cette prise de pouvoir, encore minimale ici, montre comment Fassbinder minore l'impératif égalitariste au profit d'une distribution pensée en termes de personae. En effet, il impose Schygulla non par amitié, mais parce qu'il avait eu la révélation que Schygulla allait être « un jour la star de [s]es films $»^{37}$. Sur le modèle du star system hollywoodien, le cinéphile Fassbinder recompose donc un système de positions archétypales, issues de la culture cinématographique (la jeune victime, les gangsters, les vamps...) au sein d'un collectif théâtral. Si ce système se caractérise par l'absence de hiérarchisation (pas de premiers ou de seconds rôles du fait de l'ethos scénique collectif), la distribution consistera avant tout à créer une constellation des différents archétypes, provenant de l'imaginaire cinématographique, et l'écriture à les mettre en rapport, à les disposer dans une logique plus ou moins dramatique. 

circuit des scènes institutionnelles dépendra non de l'adéquation d'un comédien à un rôle, mais de sa disposition à s'insérer dans une constellation archétypale et mythologique, cinématographique. En témoigne la mise en scène de Liliom réalisée au Schauspielhaus de Bochum en 1972. La pièce n'est pas choisie par Fassbinder, mais imposée par Peter Zadek, alors intendant de cette structure théâtrale, parce qu'elle lui semblait correspondre aux préoccupations du metteur en scène quant aux rapports intersubjectifs. Or, Fassbinder va totalement modifier la structure de la pièce pour en faire « un rêve de cinéma $»^{38}$. De fait, les costumes et les attitudes des comédiens de la production renvoient indubitablement à la mythologie hollywoodienne. Liliom n'est plus ce jeune homme, pauvre mais charmant, qui séduit les femmes par son enjouement, mais un gangster mélancolique qui traîne lentement sa lourde carcasse, accompagné par son acolyte Ficsur, à la perversité duquel il est soumis, dans une relation homosexuelle latente. Julie, sa fiancée, la petite servante innocente, devient un clone de Marylin Monroe. Quant à la tenancière jalouse du manège, c'est une diva, à la fois insensible et hystérique. Dans sa robe ceinte d'un boa de fourrure, elle n'est pas sans évoquer Greta Garbo. Les autres personnages masculins, les petits voyous de la pièce de Molnàr, deviennent des gangsters mafieux élégants portant des costumes rayés et chapeaux blancs, tout droit sortis des films noirs des années 1930. Le jeu des acteurs est lui-même défini en fonction des modèles cinématographiques. Or, la distribution des quelques personnages nommés ici reprend les choix effectués auparavant au sein de l'antiteater. Kurt Raab, abonné aux figures perverses, interprétera Ficsur, Hanna Schygulla, la star de Fassbinder, qui porte la même coiffure et la même fameuse robe que l'icône du cinéma hollywoodien des années 1950 dans Sept ans de réflexion de Billy Wilder, Julie, Wolfgang Schenck, qui avait impressionné Fassbinder lors de son passage à Brême par son impassibilité élégiaque et sa carrure à la fois puissante et fragile, Liliom, et enfin Margit Carstensen, qui collabore avec Fassbinder depuis Brême sur toutes ses productions, joue Madame Muskat, dans le même registre que lors de ces autres apparitions scéniques ou filmiques, à savoir comme une femme autoritaire au bord de la crise de nerfs. En bref, la distribution, conçue à partir du star system fassbinderien, induit une lecture particulière de la pièce, appréhendée comme le support de la manifestation d'un panthéon glamour de star, de la même manière que l'industrie cinématographique hollywoodienne générait des produits filmiques, propices à l'exhibition de personae, supports des désirs d'identification des spectateurs.

Au terme de ce parcours, il apparaît que l'emprunt à des pratiques exogènes (le cinéma ou la culture pop) est ce qui permet au théâtre de Fassbinder et de Lauwers de résoudre la tension entre l'opération de distribution et l'idée de collectif. Cet emprunt permet d'affirmer conjointement une forte auctorialité et le respect de la singularité de chaque performer. Ainsi, il nous semble qu'il témoigne d'un certain déplacement dans la pratique collective vers le modèle du groupe, où chacun des performers trouve une place qui lui est propre sous l'égide d'un auteur. D'ailleurs, la multiplication, ces dernières années, de moments pop joués live dans les mises en scène théâtrales ou spectacles chorégraphiques nous incitent à penser que la notion de groupe revêt une importance capitale dans la redéfinition d'un être-ensemble scénique, agrémentée d'une revendication de sensualité immédiate, spécifique aux genres musicaux interprétés. Par là-même, la notion de distribution, dont nous avons dit qu'elle était antinomique de celle de collectif, retrouve une nouvelle pertinence pour décrire les formes communautaires scéniques: une unité 
qui fonctionne sur la répartition des fonctions, et sur la cohabitation des personae, un phénomène pop donc.

\section{NOTES}

1. Bien plus, l'esthétique de Lauwers, son théâtre postdramatique (Hans-Thies Lehmann en fait l'un de ses représentants les plus emblématiques), sa formation de plasticien, sa pratique antérieure de la performance, sont très éloignés des productions fassbinderiennes, qui mêlent provocations politiques, épures schématiques et citations cinématographiques.

2. L'Action-Theater est une compagnie théâtrale munichoise, fondée au début de l'année 1967 par Horst Söhnlein et Ursula Strätz, avec l'intention d'expérimenter une forme de vie débarrassée de tout rapport autoritaire et d'échanges marchands, et de créer un mode de production qui refuse les traits distinctifs de la production capitaliste.

3. Le prolongement de ce parcours est, d'ailleurs, relativement semblable. Le travail avec leur collectif est relayé par la collaboration avec des scènes institutionnelles: Brême, Berlin, Francfort, Hambourg pour Fassbinder, le Burgtheater de Vienne récemment pour Jan Lauwers.

4. La constitution des ces ensembles varie dans le temps et selon les disponibilités de chacun des membres.

5. Jan Lauwers, L'Énervement, Arles, Actes Sud, 2007, p. 164.

6. Ibid., p. 167.

7. In Florian Hopf, « Meine Motivation, Filme zu machen, hat sich geändert » (1977), in Robert Fischer (dir.), Fassbinder über Fassbinder, Francfort, Verlag der Autoren, 2004, p. 372.

8. Fassbinder déclare ainsi, juste avant de mourir : «faire du théâtre avec n'importe qui, cela ne m'intéresse pas du tout » (in Bion Steinborn, Rüdiger von Naso, « Ich bin das Glück dieser Erde » (1982), in Robert Fischer (dir.), op. cit., p. 611).

9. Corinna Brocher, « Die Gruppe, die trotzdem keine war » (1973), in Robert Fischer (dir.), op.cit., p. 65-66.

10. Jan Lauwers, La Maison des cerfs, Arles, Actes-Sud, 2009, p. 54.

11. Corinna Brocher, «Die Gruppe, die trotzdem keine war» (1973), in Robert Fischer (dir.), op.cit., p. 65-66.

12. Cependant leur relégation au second plan est comme compensée par les moments chorégraphiques qu'ils assument.

13. Il faudrait ajouter le spectacle solo, créé à Avignon en 2007, pour l'actrice : La Poursuite du vent d'après Claire Goll, qui constitue, en quelque sorte, un prolongement thématique et performatif de La Chambre d'Isabella.

14. Si l'on reprend la terminologie de Georges Banu, Fassbinder et Lauwers sont à la fois «des leaders des origines ", puisqu'ils ont engendré leur collectif et « des leaders charismatiques », en ce qu'ils développent «la créativité des membres réunis" (Voir Georges Banu, «Les leaders effectifs et les communautés artistiques ", in Marie-Christine Autant-Mathieu, Créer, ensemble. Points de vue sur les communautés artistiques (fin du XIX ${ }^{e}-X^{e}$ siècle), Montpellier, L'Entretemps, 2013, p. 66-67).

15. Le film Prenez garde à la sainte putain (1971) développe beaucoup plus explicitement la problématique du rapport entre figure auctoriale et travail collectif. Seulement, Fassbinder n'incarne pas le personnage du metteur en scène, qu'il attribue à l'acteur français Lou Castel. 
Nous pouvons supposer qu'il était question trop directement du fonctionnement de l'antiteater pour que Fassbinder endosse lui-même les traits du réalisateur mettant à mort le collectif qu'il avait fondé. Il fallait de plus se dégager de l'histoire biographique pour proposer une forme réflexive sur les apories des communautés artistiques.

16. De la même manière, Jan Lauwers est présent sur scène dans les représentations de Place du Marché 76, faisant office de présentateur, de narrateur et de musicien.

17. Pieter T'Jonck, « Omdat vrouwen ontzettend belangrijk zijn. Jan Lauwers over Isabella's Room ", De Tijd, 21 septembre 2004. Cité par Felix Sprang, « Turns on the Narrative Turn. Showing and telling in Needcompany's early Shakespeare productions and Isabella's room ", in Christel Stalpaert, Frederik Le Roy, Sigrid Bousset, No Beauty for me where human life is rare, On Jan Lauwers' theatre work with Needcompany, Gand, Academia Press, 2007, p. 141.

18. Jan Lauwers, L'Énervement, op. cit., p. 162.

19. Boris Groys, «A genealogy of participatory art », in Rudolf Frieling, The Art of participation from 1950 to now, New York, Londres, Thames \& Hudson, 2008, p. 23.

20. Jan Lauwers, L'Énervement, op. cit., p. 164.

21. Arthur C. Danto, Andy Warhol, Paris, Les Belles Lettres, 2011, p. 63.

22. In programme de salle de Place du marché 76, Théâtre de Gennevilliers, mars 2015.

23. Jan Lauwers, «L'art, la philosophie et le théâtre. Entretien avec Nancy Delhalle », Alternatives théâtrales, n85-86, 2005, p. 42.

24. Voir Jan Lauwers, L'Énervement, op. cit., p. 164.

25. Entretien avec Rainer Werner Fassbinder, Donau-Kurier, 23 novembre 1971.

26. Jan Lauwers, La Maison des cerfs, op. cit., quatrième de couverture.

27. À lire les différentes déclarations rétrospectives des membres de l'antiteater, certains événements survenus lors de la vie communautaire auraient inspiré Fassbinder dans son écriture dramatique. Ainsi, Irm Hermann a plusieurs fois affirmé que la relation masochiste (dépendance affective, prostitution imposée...) qu'elle entretenait avec Fassbinder a pu influer sur le traitement des relations intersubjectives dans l'œuvre théâtrale de l'auteur munichois. Seulement, il est nécessaire de prendre des précautions avec ce genre de déclarations, étant donnée la dimension légendaire que revêtent souvent les récits ayant trait aux premières années de la bande Fassbinder. En revanche, cette interférence entre biographie et mise en récit est clairement affirmée dans l'œuvre cinématographique à plusieurs reprises (l'épisode d'Allemagne en Automne, L'Année des treize lunes, Prenez garde à la sainte putain,...), et surtout dans la mise en scène troublante de la relation du réalisateur avec sa mère, qui joue dans nombre de ses films. Notons toutefois que la pièce Les Larmes amères de Petra von Kant aurait été inspirée, selon les dires de l'auteur lui-même, par sa relation amoureuse douloureuse avec Günther Kaufmann.

28. Les différentes incarnations de chacun des acteurs semblent en effet avoir pour point commun une certaine disposition psychologique, davantage qu'une position sociale. On peut parler du sadisme de Kurt Raab, de l'hystérie de Margit Carstensen, du bovarysme d'Hannah Shygulla, de la mélancolie d'Harr Baer..., comme si Fassbinder s'ingéniait à explorer les possibles relations entre ces positions ou à les déceler dans le discours social, à travers son écriture dramatique et ses mises en scène.

29. Hans-Thies Lehmann, Le Théâtre postdramatique, op. cit., p. 175. .

30. Hans-Thies Lehmann, « Détachement. On acting in Jan Lauwers' work », in Christel Stalpaert, Frederik Le Roy, Sigrid Bousset, op. cit., p. 73.

31. Voir par exemple Richard Dyer, Stars, Londres, British Film Institute, 1979.

32. Christophe Kihm, «The Residents ou la puissance de l'anonymat », DITS, $n^{\circ}$ 6, « Rock », hiverprintemps 2006, p. 124.

33. La dernière creation de Jan Lauwers, The Blind poet, créée en mai 2015 au Kaaitheater dans le cadre du Kunstenfestival des arts exacerbe cette tendance. Celle-ci est constituée de sept portraits, les portraits des sept acteurs du spectacle, écrits par Lauwers à partir des recherches 
faites par chacun des performers sur sa généalogie, avec l'intention de montrer comment les trajectoires familiales, a priori totalement différentes, peuvent se croiser au cours de l'histoire, et par là-même, comment l'histoire est constituée de croisements entre les différentes cultures, et de mensonges identitaires. Seulement, il ne s'agit pas de véracité biographique ou familiale ici, puisque chacun des portraits, dans son actualisation scénique, tend progressivement à devenir «légendaire", au sens où il reprend et déplace les personae établies lors des précédentes productions.

34. Christophe Kihm, art. cit., p. 121.

35. Boris Groys, Portrait de l'artiste en masochiste, Paris, Arkhê, 2013, p. 42.

36. Wilfried Wiegand, «Interview I », in, coll., Fassbinder, Nantes, L'Atalante, 1982, p. 79.

37. Rainer Werner Fassbinder, Les Films libèrent la tête, Paris, L'Arche, 1984, p. 126. À partir de cette première collaboration heureuse, Fassbinder tentera, à chaque nouvelle création, d'imposer au collectif « son » actrice, bien que celle-ci n'accepte de venir travailler que si le rôle lui convient. Cette relation montre explicitement que Fassbinder suit une logique tout autre que celle du collectif politique. Il préfère le talent et le glamour de son actrice rebelle aux principes de fonctionnement du groupe.

38. Benjamin Heinrichs, "Eine Reise in den Tod - Fassbinders Theater - antiteater », Die Zeit, 18 juin 1982.

\section{RÉSUMÉS}

Les pratiques théâtrales de Rainer Werner Fassbinder et de Jan Lauwers s'inscrivent dans des contextes géographiques, historiques, esthétiques et surtout politiques différents. Pour autant, ils sont tous les deux ce que l'on pourrait appeler des auteurs de compagnie. De fait, ils écrivent leurs textes théâtraux pour une compagnie déjà constituée autour d'eux, ils écrivent pour des performers singuliers. En cela, le processus d'écriture est nécessairement informé par cette situation qui lui est inéluctablement liminaire, d'autant plus que ces collectifs sont animés par une exigence égalitariste, qui affecte la structure dramatique des textes... Écrire, c'est prendre en compte les caractéristiques physiques des performers, leurs corps, leurs voix, leurs individualités. Bien plus, l'antériorité de la compagnie constituée induit une reprise, entre différence et répétition, d'une constellation de personnages d'une pièce à l'autre, conduisant ainsi l'écriture à remettre en jeu inlassablement certaines positions subjectives et certains rapports interindividuels. L'article entend mettre au jour les caractéristiques de ces processus de création spécifiques, en pointant également les tensions qui peuvent y apparaître. Surtout, il envisage la distribution comme un phénomène pop, qui aboutit à la constitution de personae, qui rend compte de l'ambiguïté de la présence du performer, située entre authenticité subjective et archétype gestuel ou esthétique.

Beyond the historical, national and aesthetic differences of context, Rainer Werner Fassbinder and Jan Lauwers are both playwright and stage director, who work within companies (antiteater and Needcompany). They write or stage for definite performers. Therefore the precedence of the cast influences the process of creation: the physical characteristics (body, voice, and age), the compartmental ones must be taken into account. Moreover, these artists have to deal with egalitarian requirement, which necessarily affects the structure of the play. This situation implies also the repetition, with minor differences, of a constellation of characters from one 
production to the other. This article intends to analyze the specific processes of creation due to the problematic relation of an author to an ensemble with non-hierarchic pretentions, the effects of repeated scenic works with the same performers. It tries to examine it as a pop phenomenon, through the apparition of personae, these figures between fictional character and authentic individuality.

INDEX

Mots-clés : auteur, collectif, individualité, performer, persona, pop

Keywords : author, collective, individuality, performer, persona, pop 